\title{
Object Extraction in Optical Measurement Image Based on the Channel Module in Photoshop
}

\author{
LI Li-fang \\ PLA 91550 Unit 94, Liaoning Dalian, 116023, China \\ email: lilifang0427@sina.com
}

Keywords: Optical Measurement; Image Processing; Photoshop Channel; Object Extraction

\begin{abstract}
Object extracting and optical measurement of the image is researched by using the Photoshop channel module as the operating platform, and a simple and practical processing method of optical measurement image was proposed. Hereby, the optical measurement image process and the relationship between channels module and constituency is analyzed, and the method of object extraction in optical measurement image by Photoshop is studied. The image processing result shows that the object extraction based on channel module in Photoshop is an effective method.
\end{abstract}

\section{Introduction}

The application of digital image in human life is more and more widely, and digital image processing technology is more and more professional and mature. Digital image processing technology mainly includes modifying the graphics, improving the quality of the image, or extracting the effective information from the image. The mainly method of digital image processing includes: Fourier transform, the wavelet transform, various image transform, image coding and compression, using a variety of methods for image restoration and enhancement, segmentation and description and recognition of image etc. However, the above mentioned multiple methods or techniques are required for strong professional knowledge. Generally, digital staff cannot yet accomplish this work. The goal of this paper is to explore its feasibility in optical image object extraction and validate in the use of Photoshop in powerful channel function, which is in order to find a simple and practical method for optical image processing. It found in the practical application that it can be very good to complete the object extraction in optical measurement image based on the channel module in Photoshop.

\section{Photoshop technology for object extraction}

The Adobe Photoshop is a graphic image processing tool. Adobe Company launched across the PC and MAC two interfaces and it is the leading large-scale image processing software. Photoshop has many cutout methods, including the basic selection tool cutout, pen tool cutout, filter cutout, mask cutout, and set the blend mode cutout, Alpha channel cutout, channel cutout, plug-in cutout, etc. Light measuring image object of the target extraction and Photoshop cutout technology on connotation is consistent. It can pick the target up from the background, so the light measuring image object extraction is feasible by using Photoshop.

\section{Photoshop channel module principle analysis}

In Photoshop, the channel is used to store the information of image color and custom selection. Not only you can get very special districts to use the channel to aid drawing, and also you can change the color information that deposited in the channel in order to adjust image color. Whether you open a new file or scanned document, when an image is inserting into Photoshop, Photoshop can create an image file for its natural channel--members channel or color channel, the primary channel's number depends on the color of the image pattern.

Channel principle: an image of RGB mode based on red, green and blue three primary colors. In 
the channel, a figure of RGB mode, the three colors are respectively on three different channels, and each channel's color and brightness are different. Furthermore each channel is grey image base. In the tunnel, the brighter means the higher numerical value. Because of these characteristics, it can be used for the contrast of channel brightness and processing image cutout. In addition, it can also save the finished constituency parts in the channel. In the channel, white represents have and black represents have not, which is made of black, white and grey. The brightness is used to display. It also means if you want to cutout part of the image that is constituency, and then this part needs the tunnel adjusted to white.

Two important roles for the channel:

(1) Save the Constituency as channel: at the channel panel, click "save selection as channel" button, to produce an Alpha channel;

(2) Channel can save in Constituency from: in the channel panel, click "loading channel as Constituency "button.

In Photoshop among the channel, constituency and the mask have correlation each other, and the relationship is shown in Fig.1, where the arrow shows the transform.

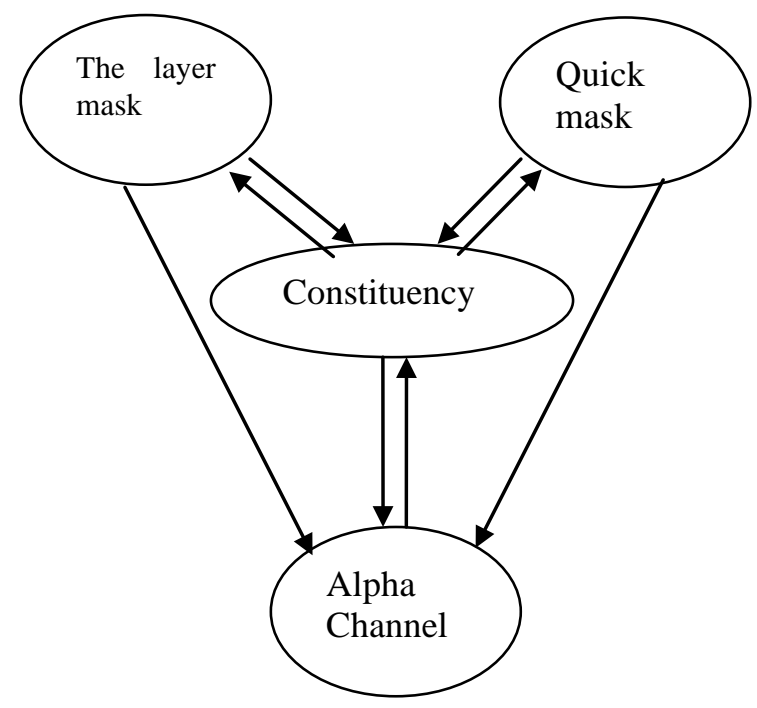

Fig.1 Relationship between channels module and constituency

Channel extraction method of concrete analysis can be summarized as follow:

(1) First analysis the image and see what are the typical features of the images, if the difference between foreground color and background color is huge, consider using channel cutout;

(2) Ensure the chosen channel, the principle to choose is to review the three channels, which channel's foreground color and background color contrast are large;

(3) After confirmation, the last step would be back up the information of the channel, in order to avoid damage during the process of dealing with the original image.

(4) By dealing with the process of channel constituency, it can also be use to increase the contrast between foreground and background colors, and curve can be used to adjust the brightness's contrast

(5) In the process, "the white represents, black represents no", if found in contrast, consider the color; if the white area is not big enough, with the "eraser" tool to erase the black area;

(6) arrived in after effects will channel into a selection, back in the layers panel;

(7) The constituency content alone on a new layer to save.

\section{Optical image object extraction by Photoshop}

The object extraction in optical image based on the channel module in Photoshop is shown in Fig. 2. 


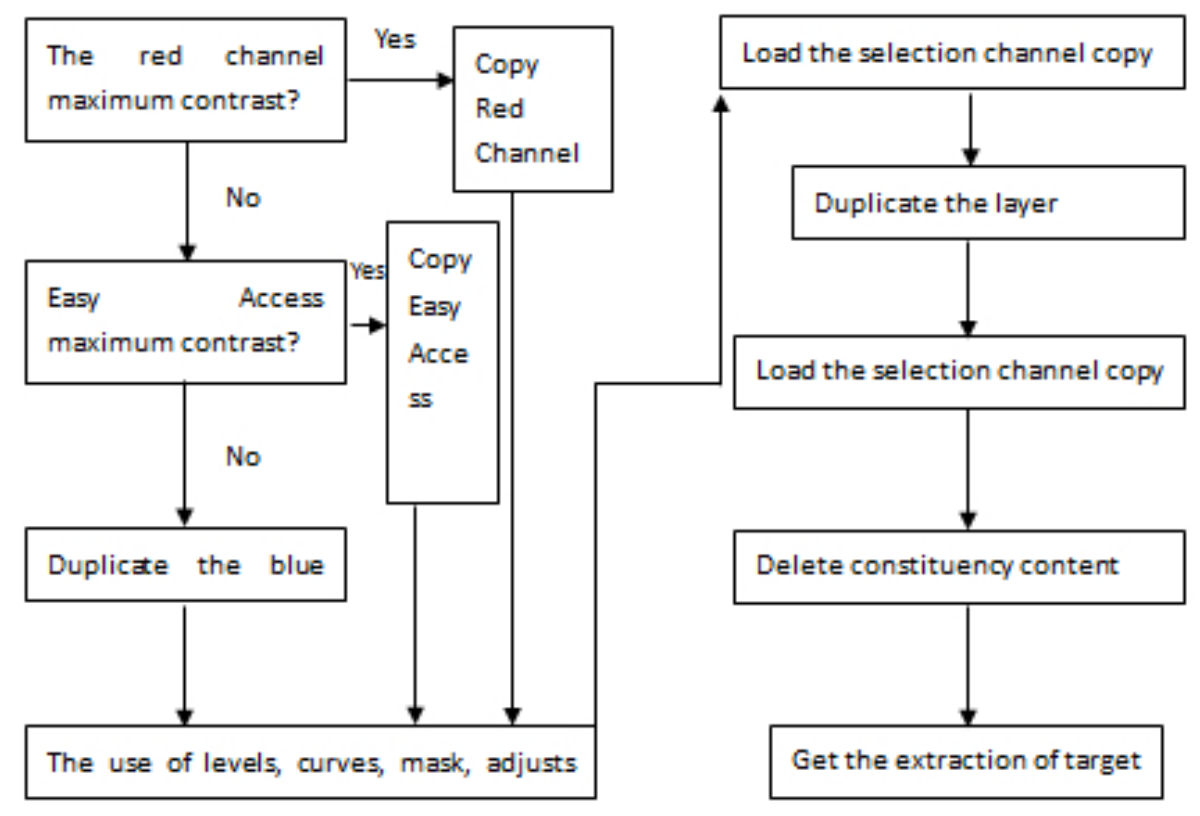

Fig.2 The process of object extraction by Photoshop

To verify the validity of the method that the object extraction based on channel module in Photoshop, the image in a plane in flight which is shown in Fig.2 is used to process, which explores the feasibility of Photoshop channels in optical image processing. The goal of this work is to extract the image of the plane.

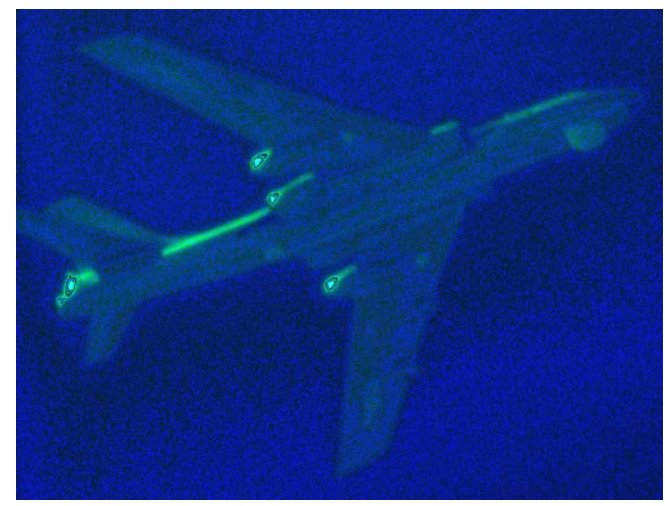

Fig.3 aircraft image

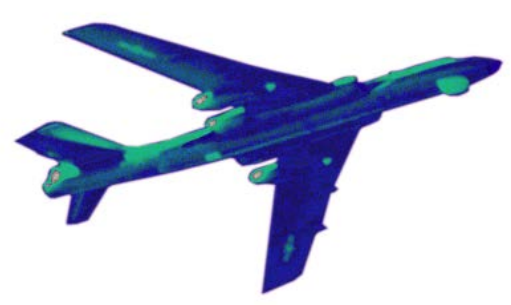

Fig. 3 extraction results

Firstly, it enters separately into the red, green, blue three channels, and then observing each channel's target and background contrast. After analysis, it can find the characteristics in the 
channel, and then we can select the largest contrast which is green channel.

Secondly, it runs "image", "adjust", "color" order. adjust curve's range, and changes the image's French grey part into a pure white. Loading red copy into constituency and hold on, and switching back to the layer mode. The next work is that copying the image and then creating a new layer 2 . Then we can paste the copy part to obtain the extraction results.

After the above processing, we can switch to the channel viewing to load the copy of red channel into constituency, keep constituency, and switch back to the layer. By this time we can delete the contents in constituency and then only layer 2 has been stroke. Through adjustment of the channel selection, density slicing reversal processing and coloring for processing, the object extraction result is shown in Fig.3. From Fig.3 can see that the aircraft's boundary is accurately be extracted, which proved that Photoshop channel module can be feasibility for target extraction in optical image measurement.

\section{Conclusion}

Through the practice of the above, this work explores the Photoshop channel module with feasibility of image object extraction. The image processing result shows that this method can be on the premise of guarantee of accuracy and largely reduce the image digital personnel work. This method has a huge advantage which does not need the deep professional background such as professional software. Photoshop makes the general digital personnel can be competent at the job. But Photoshop channel module in the optical image measurement extraction also exist some disadvantages, such as it is largely affected by the light of foreground and background image's contrast. The operating of extraction with discontinuous goal is more complex, and the accuracy would get affect. In general, if digital staff have better understanding about Photoshop software, Photoshop channel module will be very suitable for general digital personnel for optical image's measurement target extraction. Such as the layer's blending mode, channel, mask, and the conversion relationship between the selection, channel calculation principle, etc., it will largely increase the extraction efficiency and accuracy. Hope this method can be useful for the optical image's measurement of target extraction and can give great help to digital staff.

\section{References}

[1] LEI Bo. Photoshop layer and channel [M]. Beijing: China electric power press, 2008,3(2):114-231.

[2] LIN Zhao-sheng. PS matting technology [M]. Beijing: Science Press, 2008,2(1):67-85.

[3] LV Hong-gang Photoshop CS4 depth analysis [M]. Beijing: weapon press, 2010,2(4):108-178.

[4] Digital creative. Layer, channel, mask the depth analysis [M]. Beijing: Publishing House of electronics industry, 2009:1-432.

[5] Mark F. Photoshop recovery and modified [M]. Beijing: Tsinghua University press, 2009, 3(2):176-213. 